\title{
THE MANAGEMENT OF SCOLIOSIS
}

\author{
By J. I. P. JAMES, M.S., F.R.C.S. \\ From the Scoliosis Clinic, The Roval National Orthopaedic Hospital and Institute of Orthopaedics, London
}

In the early days of orthopaedic surgery, when operations were simple procedures and our predecessors were skilled in the design of surgical appliances, scoliosis was one of the deformities which occupied much of their attention. As surgery improved, diseases more amenable to operative treatment became the centre of interest. Though a few men continued their work on scoliosis along traditional lines, most orthopaedic surgeons lost interest in a condition which seemed to respond so rarely, and scoliosis often went untreated except for the most perfunctory measures. That this should happen was not unnatural; scoliosis had proved a hard nut to crack and the methods used did not appeal to the surgeon of the operative school. However, the real cause of this neglect lay deeper, for most men, though lacking proof, felt that the methods available were useless. In addition, there were no basic methods of studying the disease. The position is now altered and we may reasonably claim that after many years of attack certain types of scoliosis are showing signs of yielding, though with nothing like unconditional surrender.

One of the most important advances has been the development of accurate measurement of the curves, by the method of Ferguson (1930), later modified by Cobb (1948). By this means it has been possible to follow the evolution of the deformity and to assess the results of treatment. On all conservative methods, such as plaster jackets, spinal braces, long continued head suspension, special systems of exercise, and so forth, the verdict has been a striking one. In 194I the American Orthopaedic Association published the report of a committee investigating such conservative treatment in most of their large clinics: in not a single instance had a curvature decreased during treatment.

Spinal fusion as a method of treating scoliosis was first employed by Hibbs. Risser, of the same hospital, later developed his correcting turnbuckle jacket, still the most effective method of correction. Correction and fusion by their methods were used with enthusiasm in the years after the first world war. Disquiet soon arose, however, because correction was commonly lost on account of pseudarthrosis; it was also sometimes found that $\infty$ although the primary curve could be successfully corrected, rigid compensatory curves remained unable to 'decompensate' and the patient listed badly to one side. Cobb, however, modified the technique of operation and thereby obtained a higher fusion rate. At the same time he developed the use of the 'bending film,' a fundamental radiological investigation which enables us to $\vec{\oplus}$ estimate the maximum permissible correction of on the primary curve in relation to the mobility of the compensatory curves. The use of this film will be detailed later.

It is interesting to pause a moment and see how the influence of one hospital has transformed the treatment of this disorder. As already stated, Hibbs, of the New York Orthopaedic Hospital, was the first surgeon to treat scoliosis by fusion. Risser, Ferguson, Von Lackum and Cobb have all worked at the same hospital and it is these men who have so greatly advanced our knowledge. $\mathrm{Up}$ to the last few years, indeed, they were responsible for all the major advances.

Until quite recently, although some of the causes of scoliosis were understood, the natural history of none of the types had been fully worked out, least of all in the commonest, the idiopathic type. Then in April 1950, Ponseti and Friedman, of Iowa City, published an article of the greatest importance, showing that in idiopathic scoliosis the prognosis is related to the site of the apex of the primary curve. It is thus possible to predict how such a curve will behave and what problems may develop. Here is the key to the most important variety of this complex deformity.

Armed now with a method of curve measurement, a better understanding of prognosis and a means of correction and fusion, we may proceed to discuss the management of scoliosis. 
As in all diseases, an exact diagnosis and an understanding of the aetiology are essential.

\section{Clinical Classification}

Scoliosis is a lateral curvature of the spine. There should be little difficulty in deciding whether it is present or not. When a lateral curvature is discovered it is essential to decide whether it is postural or structural.

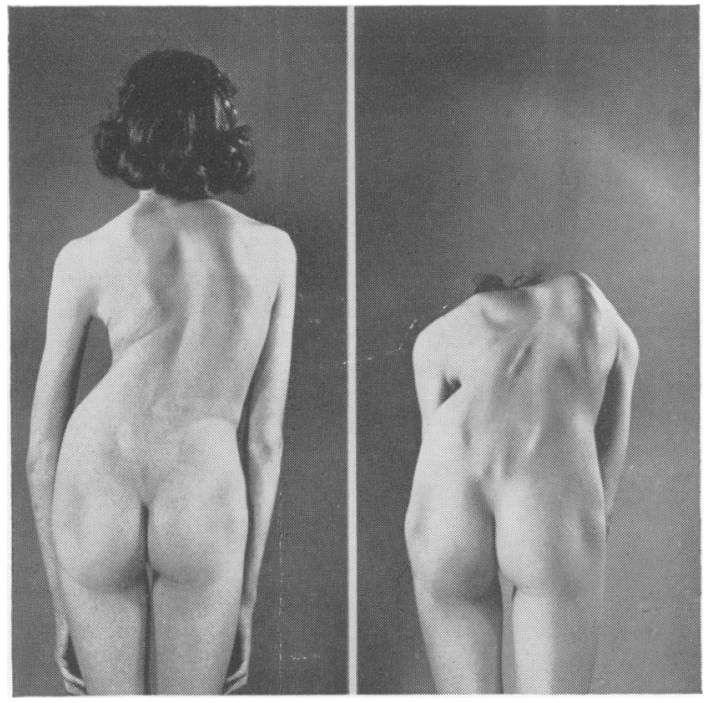

FIG. 1.-A girl with a triple curve primary thoracic idiopathic scoliosis. Note that only the primary curve shows rotation and that this remains on forward flexion.

\section{Postural Scoliosis}

Postural scoliosis is a single lateral curvature of slight degree which disappears on suspension or on bending forward; in the latter position no vertebral or rib rotation is seen. Postural scoliosis then is a lateral curvature without rotation, this distinction from structural scoliosis being fundamental. It disappears spontaneously or with simple exercises and does not change to a structural curve. It has long been a misconception that it was the earliest sign of a curvature which might become serious.

Compensatory scoliosis, due to a short leg or to a deformity of the hip joint, can always be excluded by a careful initial examination. It shows two long curves without rotation, commencing at the lumbo-sacral junction. Compensatory scoliosis does not appear to progress and has no great practical importance; it is rarely, if ever, a fixed structural curve. The treatment is entirely that of the primary cause.

\section{Structural Scoliosis}

Structural scoliosis is characterized by the presence of a lateral curvature with rotation. The lateral curvature itself does not disappear on suspension or with forward flexion of the spine, and the vertebral or rib rotation persists in all positions (Fig. I).

The primary cause of a structural scoliosis may sometimes be identified with ease. Infantile paralysis, congenital abnormalities and neurofibromatosis are in this group. In addition there are many rare causes, for example muscular dystrophy, neuropathies such as syringomyelia, spastic paralysis and Friedreich's ataxia, rickets (a rare cause in Great Britain nowadays) and some other conditions where the relationship is not clearly understood. This leaves the main group of idiopathic structural scoliosis for consideration. As the term implies, no reasonable explanation exists for this condition, and no published work has advanced our knowledge of the possible aetiology.

\section{Idiopathic Scoliosis}

The onset is at any age during childhood. The deformity tends to increase rapidly during periods of rapid growth, but does not always do so; many cases in fact become arrested before growth has ceased, and for this there is no obvious explanation. In general terms, the earlier the onset the more serious the prognosis, because the curvature has a longer time to progress and because both the primary and compensatory curves in time become rigid, making correction difficult. In infancy males predominate, but in the early teens, the commonest age of onset, girls are affected about fifteen times more often than boys. The adolescent who develops scoliosis is usually a robust and otherwise normal child. As mentioned earlier, Ponseti and Friedman have recently found that the site of the primary curve is of great importance in prognosis; the higher the curve, the worse the prognosis.

In idiopathic scoliosis there are either three or four curves, best seen in the radiograph. When there are three, the middle curve is primary, with a compensatory curve above and below. At first only the primary curve is accompanied by clinical rotation; later the compensatory curves may also acquire some fixed rotation. When there are four curves the middle two are primary, with small compensatory curves above and below; both primary curves then show marked rotation.

While considering aetiology and prognosis it is convenient to discuss an observation made by Risser. He noted that idiopathic scoliosis ceases to progress when the iliac apophyses appear in the 


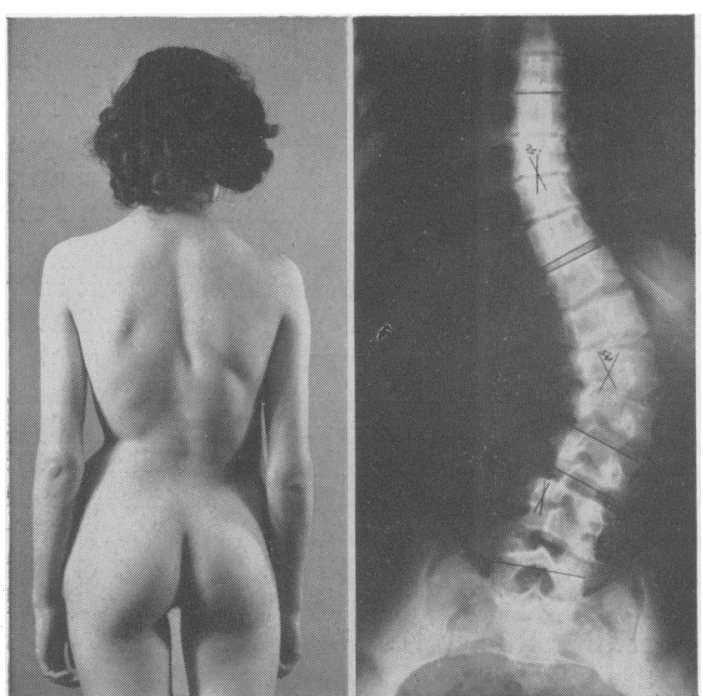

FIG. 2.-Primary lumbar idiopathic scoliosis. The curve has ceased to progress at $54^{\circ}$. Though the curve is rather more severe than usual the deformity is slight.

radiographs all the way round the crests from the anterior to the posterior superior spines. This complete appearance of the iliac apophyses is presumably an index of skeletal maturation and coincides with fusion of the vertebral epiphyses. It is a variable event in terms of the age in years, but in practice it is a valuable end-point in this deformity of the growing child. In girls the onset of menstruation usually precedes the full'development of the apophyses by some months.

\section{Curve patterns in Idiopathic Scoliosis}

\section{Lumbar Idiopathic Scoliosis (Fig. 2)}

In this common curve pattern the primary curve is in the lumbar region with its apex at L.I, 2 or 3. Because the curvature is slight and because no ribs are involved in the rotation, deformity is always minimal. It never becomes severe and the curve is seldom as much as $80^{\circ}$. This type therefore never requires correction and fusion, and this opinion may be confidently given from the earliest stages of its development.

In later life, because of the rotation of the posterior joints in this mobile area, a degenerative osteo-arthritis often develops and a fairly high proportion of patients with this pattern suffer backache. Pain is not a common symptom in scoliosis and is largely confined to this group. It may be relieved by a corset, otherwise by fusion without correction.

\section{Thoraco-lumbar Idiopathic Scoliosis (Fig. 3)}

This less common pattern has the apex-of the

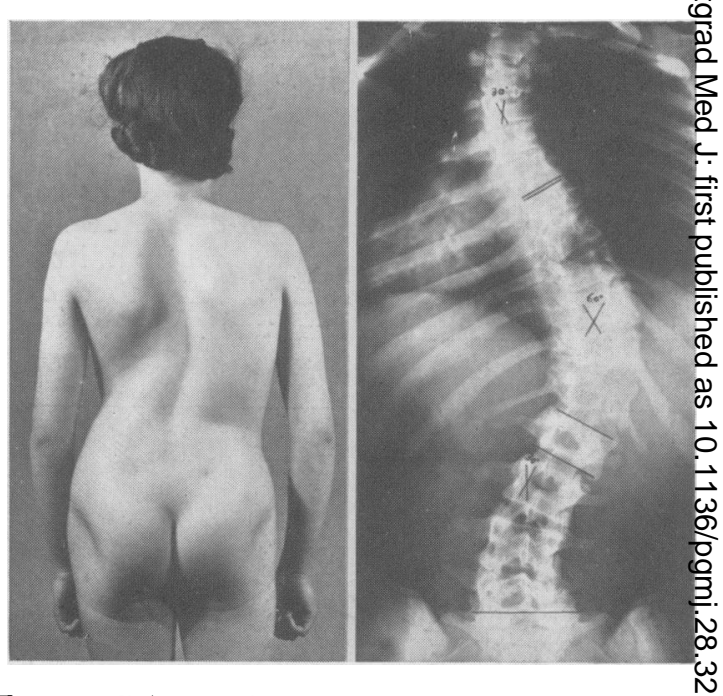

FIG. 3.-Primary thoraco-lumbar idiopathic scoliosis The apex of the curve is at T.II or 12 and the prognosis is good. This is a fairly typical en $\Phi$ result.

curve at T.I I or 12. As will be seen, it is inter mediate in type between the lumbar and thoracic patterns. The prognosis is good, with rare excepe tions which may require correction and fusion

\section{Thoracic Idiopathic Scoliosis (Fig. 4)}

This is a common and important pattern. Only 5 to 10 per cent. of all cases of idiopathic scoliosis become sufficiently severe to warrant correction and fusion, and it is almost exclusively in this thoraciG. group that such cases are found. The prognosis is poor because of the severity of the curvature which may reach $15^{\circ}$, and because the involve ment of the ribs in the rotation leads to an obvious deformity. In adolescence, girls are more ofteng affected and the curve is more often to the right:-

Infantile Idiopathic Thoracic Scoliosis. When. idiopathic scoliosis starts in infancy, it occurs predominantly in boys and the curve is usually to the left; except in rare instances the primaryo curve is situated in the thoracic region. The deformity starts between six months and three years after birth, which suggests that it is unrelated. to moulding from intra-uterine position. Because of its early onset and thoracic location, the prog 0 nosis is serious. There are many exceptions however; some cases remain static and a few of minimal degree may even disappear spontaneously

Combined Thoracic and Lumbar Idiopathic Scoliosis

(Fig. 5)

This pattern is the only quadruple curve idiopathic scoliosis. The age of onset is some $\stackrel{\mathbb{P}}{?}$ what younger than the average. The adjacent 


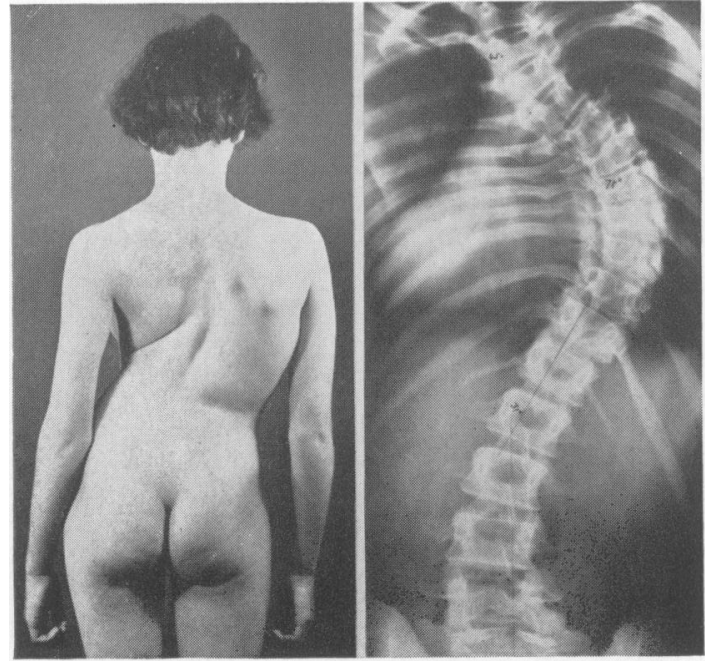

FIG. 4. Thoracic idiopathic scoliosis of moderate degree. This common pattern frequently has a severe prognosis. It leads to marked deformity because of the dropped shoulder and prominent hip.

lumbar and thoracic curves are both primary with severe rotation, and the two curves progress at roughly the same rate. Despite the frequency of severe curvature and of radiographs that look grotesque, this pattern has a good prognosis. In marked contrast with the thoracic pattern, the shoulders remain level and the hip is covered; because each posterior rotation balances the other, the clothes hang well.

Correction and fusion of this pattern is a formidable procedure because two primary curves in opposite directions need correction and fusion. A Risser jacket is unsuitable and the Von Lackum transection jacket is used. The problem fortunately is not a common one.

\section{Paralytic Scoliosis}

After an attack of poliomyelitis with residual paralysis of trunk muscles in a growing child, the development of a structural curve is a common event. These cases have characteristic curvatures, quite distinct from idiopathic scoliosis; moreover, the sex distribution is equal. Such observations are sound arguments that the idiopathic variety is not due to unrecognized poliomyelitis.

Paralytic curves fall into two main groups. The collapsing spine is associated with severe and symmetrical paralysis; no severe fixed curve develops, but the spine collapses because it is unstable in the erect position. In the second and larger group the curve develops from asymmetrical weakness of trunk muscles. The convexity of the primary curve is on the weaker side; the curve

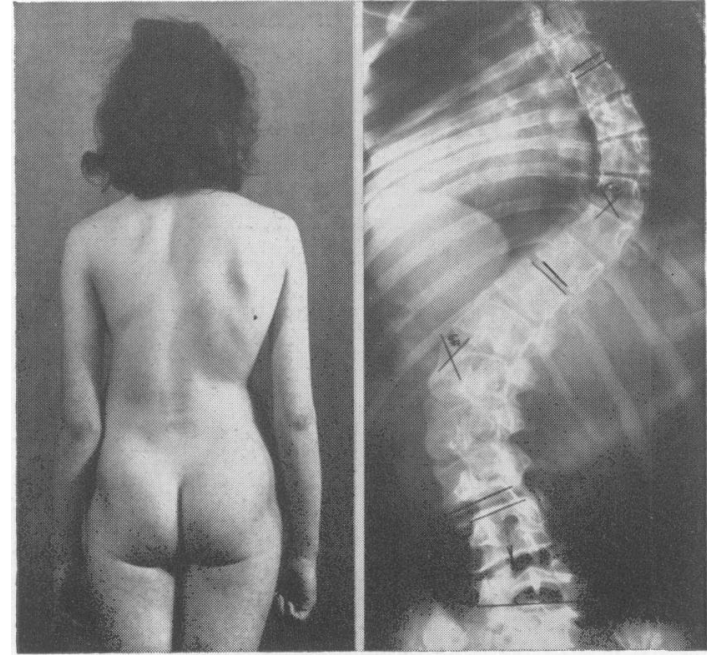

FIG. 5.-Combined thoracic and lumbar idiopathic scoliosis. Two primary curves and two compensatory curves above and below are present. The primary curves are almost equal. Despite the marked radiographic changes the appearance is better than that of the patient shown in Fig. 4.

soon shows rotation and is usually progressive, often to a serious degree. Three types may be defined, thoraco-lumbar, low thoracic and high thoracic, but these patterns are not so well defined as in idiopathic scoliosis because the paralysis is so variable.

Thoraco-lumbar paralytic scoliosis is usually associated with weak lateral abdominal and quadratus lumborum muscles, a low thoracic curve with weak intercostals and lateral abdominals on the one side. Being low and easy to hinge out,

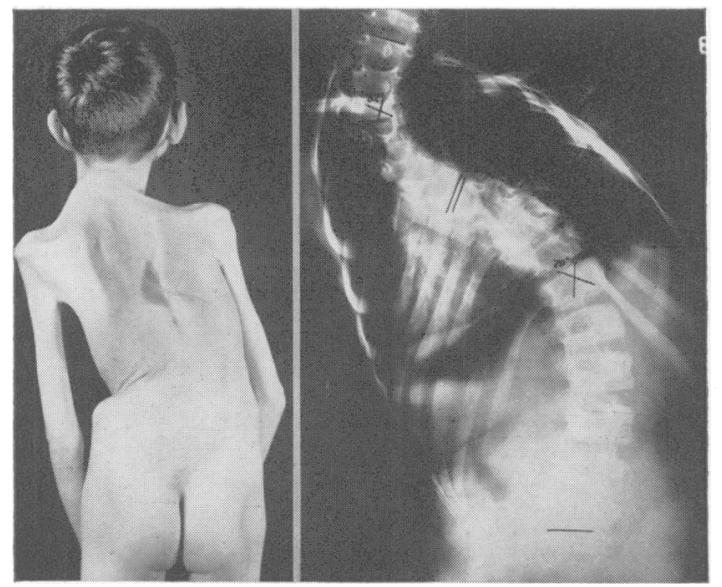

FIG. 6.-A high thoracic paralytic curve of $82^{\circ}$ extending from T.I to T.7. The deformity is probably due to paralysis of the intercostals on the left side. 
these curves are eminently suitable for correction and, being paralytic in origin, the curves are relatively mobile.

The high thoracic curve is notoriously serious (Fig. 6). It is associated with unilateral weakness of the intercostals and extends caudally from the level of T.I. It produces a particularly ugly deformity, because the upper compensatory curve is in the neck; in consequence, the head seems to be 'stepped sideways' on the trunk. It is too high for correction and requires early fusion before a severe deformity develops.

No accurate prognosis can be given in paralytic scoliosis owing to the difficulty of grading the affected muscles and because of the complexity of the curve patterns. As usual, an early onset is more serious. Muscle imbalance is more significant than the actual severity of the paralysis.

\section{Congenital Scoliosis}

Many types of bony abnormality may occur in the spine, but they are so variable that a prognosis based on the radiological picture is impossible. Frequently a spine with numerous abnormalities, for example several hemivertebrae, will show less curvature than one with a single abnormality, because the effect of one wedge may cancel the other. An abnormality occurring at the lumbosacral junction may cause severe curvature; because there is no room below for a compensatory curve, a double curve develops, the lower of which is primary. Apart from compensatory scoliosis, nearly all double curves are congenital.

\section{Neurofibromatosis}

A material proportion of cases of von Recklinghausen's neurofibromatosis develop scoliosis, sometimes with paraplegia. The curve is usually short, thoracic and acute. In such cases a careful search should be made for pigmented areas, which are often overlooked in the examination of scoliosis (Fig. 7). The curvature is almost certain to progress; no other type of scoliosis has such a bad prognosis.

\section{Case Records of Scoliosis}

It is our practice at the patient's first visit to make a thorough examination, including a complete check of muscle power; every effort is made to determine the aetiology. Where the aetiology has been discovered, or by exclusion a diagnosis of idiopathic scoliosis has been made, the curve pattern is next established from radiographs taken, if necessary, in both the erect and the supine positions. All the curves are measured and an assessment of the prognosis is recorded.

The measurement of the angles of curvature is quite simple (Fig. 3). The limit of each curve

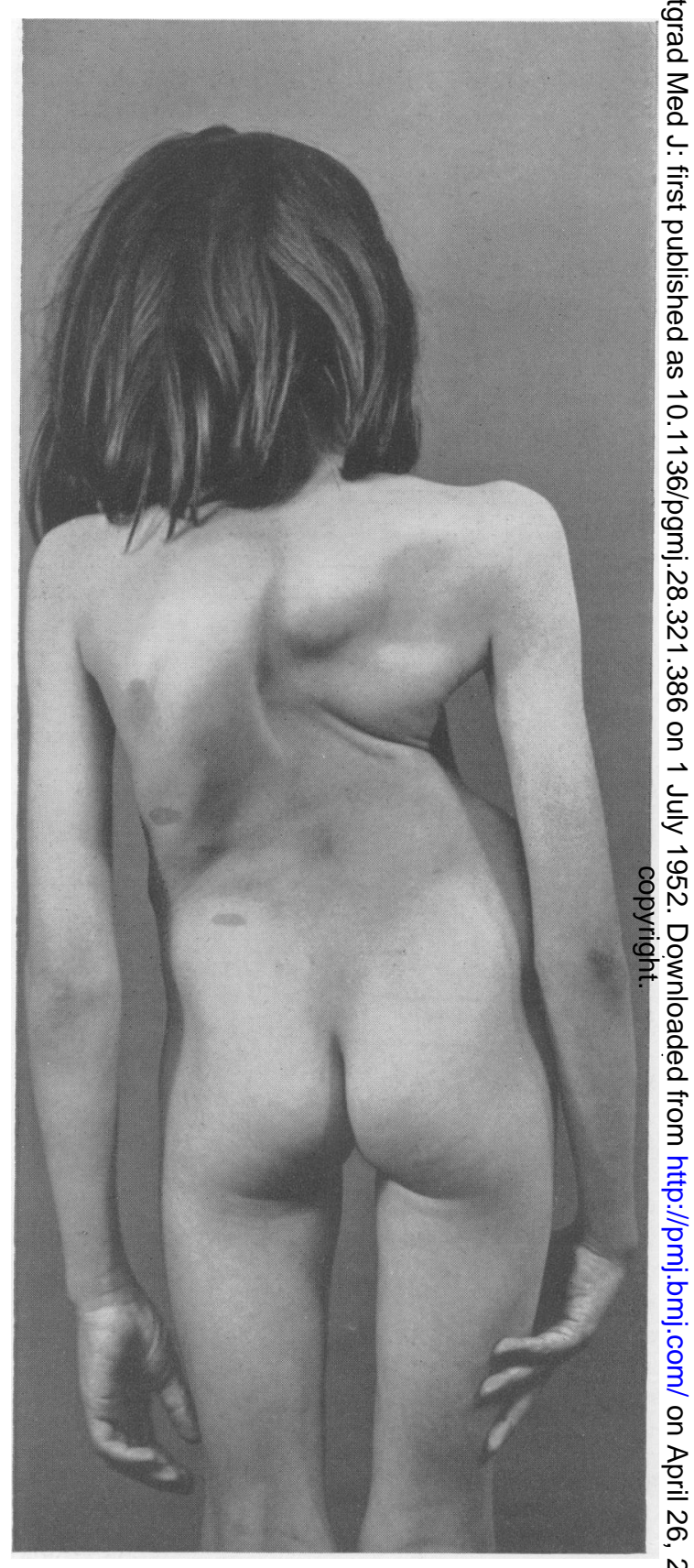

FIG. 7.-A patient showing the typical café-au-lait $\mathrm{N}$ patches of neurofibromatosis over the left side of ${ }^{\omega}$
the chest.

can be distinguished by noting that, whereas in the primary curve the disc is widened on one side, $\stackrel{?}{+}$ in the curve above or below it is widened on the $\frac{T}{0}$ opposite side. At each junction there is a neutral $\underset{\mathbb{D}}{\circ}$

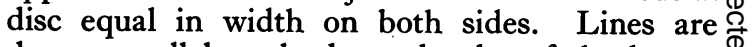
drawn parallel to the lower border of the lowest $\stackrel{\varnothing}{\circ}$ 


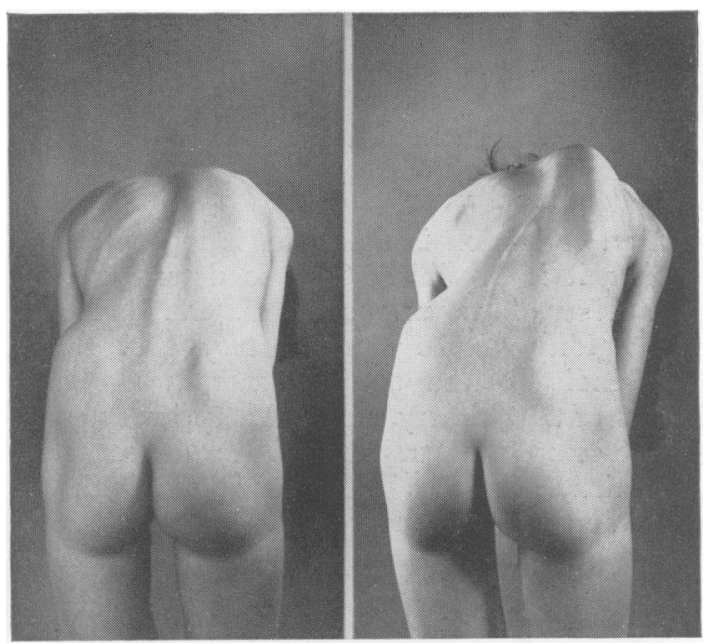

FIG. 8.-Photographs of a girl with a right thoracic structural scoliosis taken at an interval of one year. The earlier photograph shows a slight curvature to the right with moderate but quite definite rotation of the ribs. A year later both the curvature and the rotation are much more obvious. Note that rotation is absent from the lower compensatory curve in both views.

vertebra of each curve and from them perpendiculars are erected. Similar lines are drawn at right-angles to the upper border of each highest vertebra. The angles at which each pair of perpendiculars meet are the angles of curvature. The measurements for both primary and compensatory curves are recorded.

Thereafter at intervals of three to six months the patient, usually a child, attends for observation and radiographs. At each visit the curves are measured and any deterioration noted (Fig. 8). Occasionally the curve may have increased to such a degree that correction and fusion is indicated forthwith, but in the vast majority of cases regular observation is all that is required. These records are of great value in accumulating data on the natural history of the various types, of many of which our knowledge is still very incomplete.

\section{Treatment of Structural Scoliosis}

The modern treatment of scoliosis is based on the observed behaviour of the various types and patterns of curvature. Some are known to have an excellent prognosis with or without treatment, whereas others present difficult problems. With our knowledge of prognosis we can at the beginning either warn parents of future trouble, or more often reassure them.

We have already seen that structural curves are not improved by conservative treatment, and it seems unlikely that we can even arrest the progress of the deformity by such measures. Though suitable exercises may, of course, improve the posture and appearance and must help to retain the mobility of the curves, we should not deceive either ourselves or parents into thinking that we can obtain correction or even arrest by such means. If exercises are to be used, their limited value should be remembered and frequent attendance at hospital to the detriment of schooling or at expense and inconvenience to parents should be avoided.

Spinal supports, plaster jackets and plaster beds should be prescribed with caution. These unpleasant encumbrances achieve very little and favour rigidity of both primary and compensatory curves. In general it is our practice to use no conservative treatment of this type, but in the treatment of paralytic curvature certain exceptions are made. Exercises to improve the power of partially paralysed muscles are logical, though physiotherapy will seldom improve muscles after the first eighteen months or two years if they have been used in everyday activity. Spinal supports, on the other hand, are occasionally needed. Often with partial symmetrical paralysis of the trunk muscles, and always with total paralysis, there is a general collapse on becoming erect. Therefore some of these patients are unable to walk unless they wear an efficient spinal support.

\section{Indications for Correction and Fusion}

Scoliosis becomes a crippling deformity in a minority of cases; much of the disability is cosmetic, though this is not unimportant, especially in young girls. Correction may obviously be indicated when the deformity is ugly, or the disability sufficient to justify a long and major surgical procedure. In deciding whether correction is advisable for cosmetic reasons, it must be realized that while depression of the shoulder and prominence of the hip can be corrected, the projecting rib hump responds only to a small degree. More important in practice is the young patient with moderate deformity in whom the prognosis clearly indicates continued deterioration. In our present state of knowledge correction and fusion is unwise before the age of ten years, but the early case is easier to treat and more rewarding than the late neglected case with marked bone changes and soft tissue contractures.

In idiopathic scoliosis almost the only pattern requiring correction is the thoracic triple curve. In the mature child it is generally found that a curve of $65^{\circ}$ to $80^{\circ}$ is ugly enough to warrant correction, though the appearance of the patient is more important than the radiographic measurement. For a girl aged ten years with a $55^{\circ}$ or $60^{\circ}$ curve of this pattern, correction would be indicated, 
having regard to the future rather than the present curvature.

Paralytic scoliosis is frequently severe and a considerable proportion of cases require correction. The thoraco-lumbar and low thoracic curves need correction when a severe deformity already exists, or when there is considerable lateral muscular imbalance in the young child and marked deformity can be expected. The upper thoracic curve is too high for correction and fusion is necessary in the position of deformity at the earliest opportunity. Its discovery before the age of ten years may occasionally lead the surgeon to consider fusion at an earlier age; whether this may be safely done has still to be determined.

The flail or collapsing spine due to severe symmetrical paralysis presents different problems. Here we are not concerned with either the prevention or correction of severe scoliosis; it will not occur. The aim is a rigid spine linking the legs to the upper trunk in a stable fashion. This entails a fusion from sacrum up to perhaps the mid-thoracic region. Before undertaking this formidable procedure one point must be settled beyond doubt. If the patient can walk it must be determined whether the hip flexors are strong enough to allow walking with the spine fixed to the pelvis, or whether the lateral abdominal muscles are used to elevate each side of the pelvis in turn, so that the legs can swing through. If the latter method of progression is used, fusion of the lumbar spine to the sacrum will effectively stop all walking. When in doubt, the application of a well-moulded plaster spinal jacket which imitates the effects of arthrodesis will settle the matter.

A few patients who can walk laboriously in double calipers and a spinal support, and who cannot sit up without such a support, are worthy of fusion. Although walking may be impeded, they become 'good sitters.' The arthrodesis of the spine holds them rigidly upright, so that they can sit at a bench or a typewriter and use both hands.

We have now briefly to consider the large group of children under the age of ten years with severe scoliosis of any aetiology other than anterior poliomyelitis. It must be emphasized that from the evidence available nothing can be done to arrest a progressing curve except operation. In infants, plaster beds are still under trial, but the results to date are unimpressive. In general, therefore, the deformity is allowed to run its course until fusion is possible at the age of ten.

In the rarer types of scoliosis, the indications for fusion vary considerably. In neurofibromatosis it is unusual for a case not to require correction, as the prognosis is exceedingly bad. In con- genital curvature, the prognosis is not easily assessed, but is frequently good; constant observation with correction and fusion when needed is the present policy.

\section{Mobility of the Curves}

When correction is desirable, it must be seen whether it is possible. This is determined by the mobility of the various curves. Paradoxically, it is rigidity of the compensatory curves that contra-indicates the attempt more often than rigidity of the primary curve. If a primary curve is to be completely straightened in a Risser jacket, the patient must be able to straighten both the upper and lower compensatory curves in order to bring the head over the pelvis. If the compensatory curves are rigid, this will not be possible and the patient will look like the leaning tower of Pisa. In earlier years over-correction of the primary curve was often produced with such disastrous results.
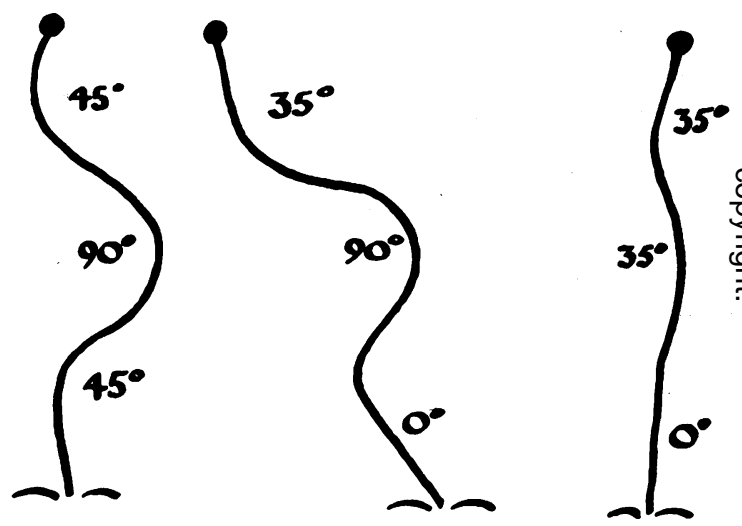

Fig. 9.- Line drawings to demonstrate the bending film. The first drawing represents the anteroposterior view taken with the patient erect. The second drawing illustrates the effect of bending towards the side of the convexity of the compensatory curves; the lower compensatory curve has straightened completely, but the upper one only to $35^{\circ}$. The third drawing shows how after correction and fusion of the primary curve to $35^{\circ}$ the patient should be able to straighten the trunk and be left well balanced with two curves of $35^{\circ}$ in either direction.

The Bending Film. The amount of fixed $\stackrel{N}{N}$ rigidity of the compensatory curves is determined $\mathrm{N}$ by the bending film, which is an antero-posterior view, taken with the patient standing and bending $O$ to the side of the convexity of the compensatory $\Phi$ curves, usually to the left. If the primary curve is of recent origin, and particularly if paralytic, these compensatory curves may straighten out completely. It is then permissible to straighten the primary curve completely. If the patient is 


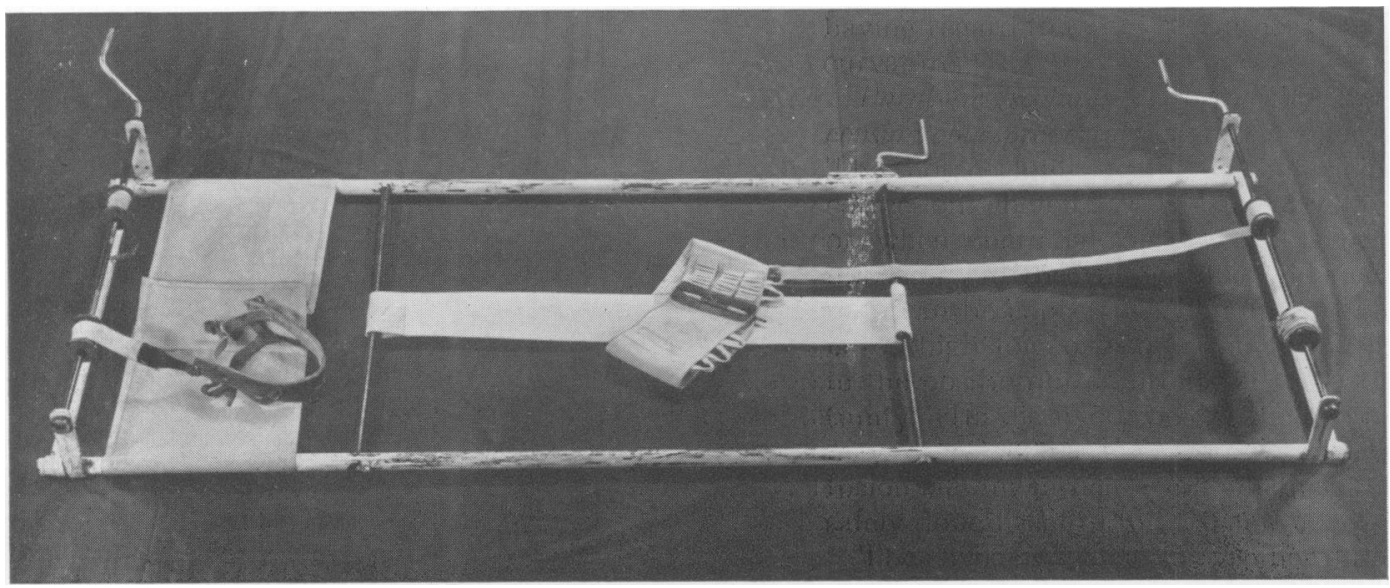

FIG. 10.-The Risser frame. Stockinette is first applied over the patient's trunk and one thigh. The patient lies on the long canvas strap which is detached at its upper end by sliding out the transverse rod and introduced under the stockinette from the back of the neck to the perineum. In certain cases the head halter and the pelvic girdle are used to apply distraction to the trunk. Both the canvas belt and the pelvic girdle may be removed with ease after application of the jacket.

an adult or the scoliosis is of long standing, however, the compensatory curves may be virtually unaltered by bending. In this rather unusual circumstance any correction is over-correction.

The usual finding is somewhere intermediate between the two (Fig. 9). For instance, in a triple curve scoliosis with a primary curve of $90^{\circ}$ and compensatory curves above and below of $45^{\circ}$ each, it might be found that the lower curve straightened out but that the upper curve straightened only to $35^{\circ}$. It would then be permissible to correct the primary curve from $90^{\circ}$ to $35^{\circ}$, that is a correction of $55^{\circ}$. In the end such a patient would have a primary curvature of $35^{\circ}$ to one side and an upper compensatory curvature of $35^{\circ}$ to the opposite side; the head would therefore be over the pelvis and the patient well balanced.

The Tilt Film. One further examination of the paralytic curve is to determine whether or not the lower compensatory curve can be held to the full extent of its mobility by the weakened muscles acting against gravity. This is determined by a tilt film, the sitting patient being tilted off the vertical by a 3 -in. block under whichever buttock is on the side of the convexity of the lower compensatory curve. From this position he attempts to bring himself vertically in front of the $\mathrm{X}$-ray screen by actively obliterating the lower curve. If he cannot do so, the fusion may have to be extended down to the sacrum, provided of course that the flexors of the hips are adequate.

\section{Technique of Correction}

Correction of the primary curvature is achieved by the use of the Risser turnbuckle jacket, the most effective method yet devised. For the 'flail' spine when no correction is required and for the high thoracic paralytic curve where none is possible, the patient is simply put into a plasteg bed or a plaster spinal jacket with a posteriop window before operation.

The Risser jacket is applied on the speciat frame of that name (Fig. Io). It is a full spinal jacket with shoulder straps; the leg on the side of the primary curve is included down to the knee; no head piece is now used (Fig. II). Anterior and posterior hinges are placed on the side of the convexity, well lateral and opposite to the apex of the primary curve; on the concave side is the turnbuckle screw. When the plaster is thoroughly dry it is cut transversely at the level of the apex of the curve. A small horizontal ellipse is removed on the side of the convexity between the hinges. The jacket is then ready for correction to begin.

Correction by elongating the turnbuckle is rapid at first, but slows as the more rigid part of the deformity is reached. Discomfort should never be produced. A mobile paralytic curve may correct in two weeks, whereas a rigid idiopathic curve may take ten weeks. Radiographs are taken when correction is nearly complete and repeated until the desired amount is obtained. In the younger age group it is nearly always possible to achieve this, but in older patients or patients with scoliosis dating from infancy, correction may fall short of the degree thought possible from the bending film. It is found that this end-point of correction can be judged, because the patient tends to slip out of the Risser jacket instead of being further straightened. Although 


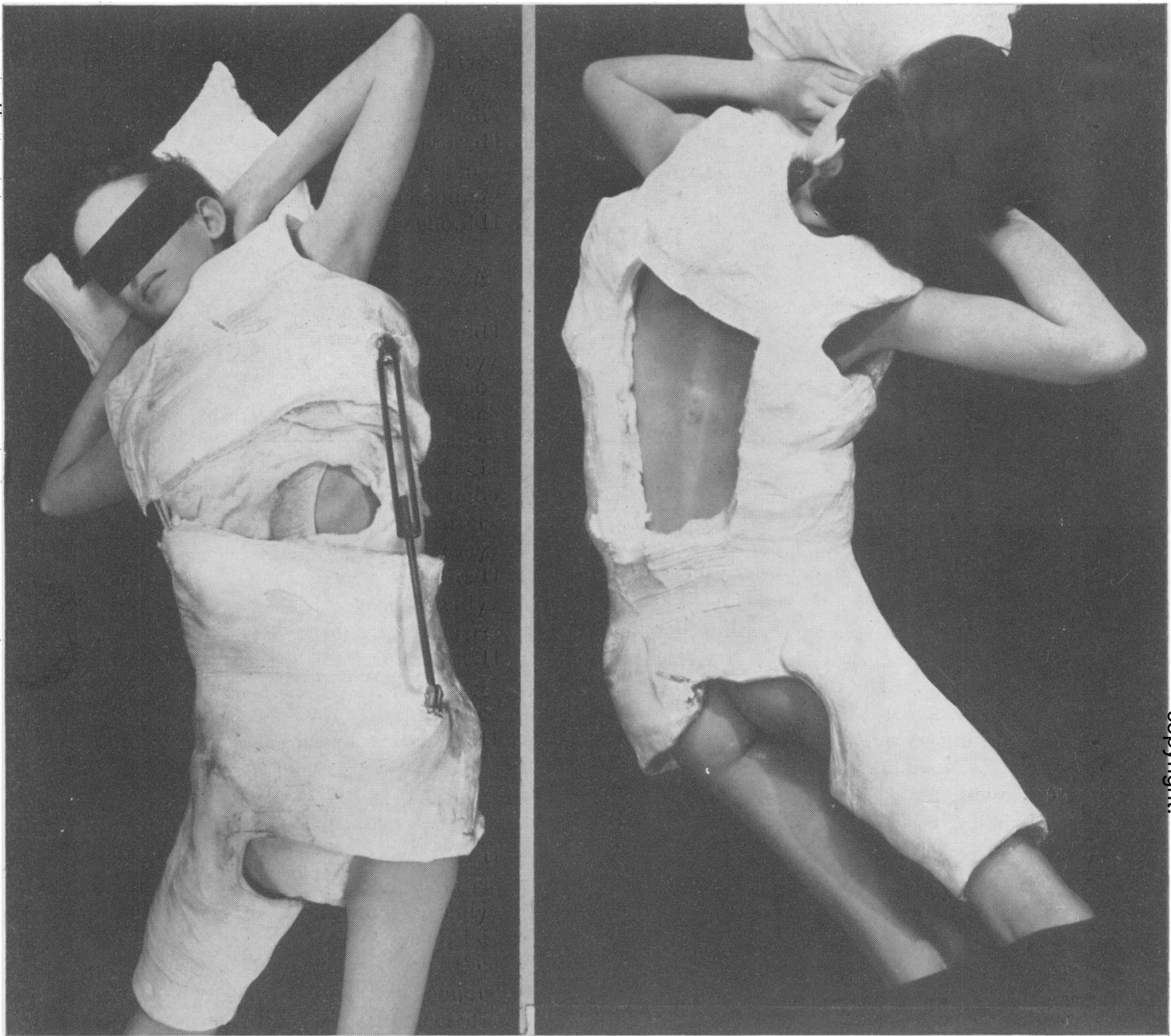

FIG. II.-A Risser jacket applied for the correction of a right thoraco-lumbar laralytic curve. In the second figure the plaster has been closed after correction and a window cut posteriorly to allow access for spinal fusion.

it is possible that a primary curve might prove so rigid as to be uncorrectible despite a satisfactory bending film, this has not occurred in practice. In our personal cases operation has not yet been refused on account of a lack of correction in a patient with good bending films, though the correction has often been less than that desired.

With careful nursing, complications seldom arise, apart from minor pressure sores. Transient nerve paralysis is of two types. A 'Saturday night' paralysis is caused by the arm lying over an edge of the plaster cast during sleep. A few transient brachial plexus lesions have been of the upper trunk C.5 and 6, not traction lesions of the lower trunk as might have been expected. They are probably due to compression of the plexus between the shoulder girdle and the side of the neck.

When correction is finished, the wedge-shaped space opened out in the plaster on the side of the turn-buckle is closed. Later a window is cut over the spine to allow access for operation (Fig. I I). A marker is placed over one spinous process, a radiograph is taken and a known vertebral level obtained.

\section{Technique of Spinal Fusion}

The aim of the operation is to obtain a wide and deep graft soundly fused to all the vertebrae; anything less than this leads to relapse. The method of fusion is simple in principle, but meticulous and tedious in practice. The extent of 


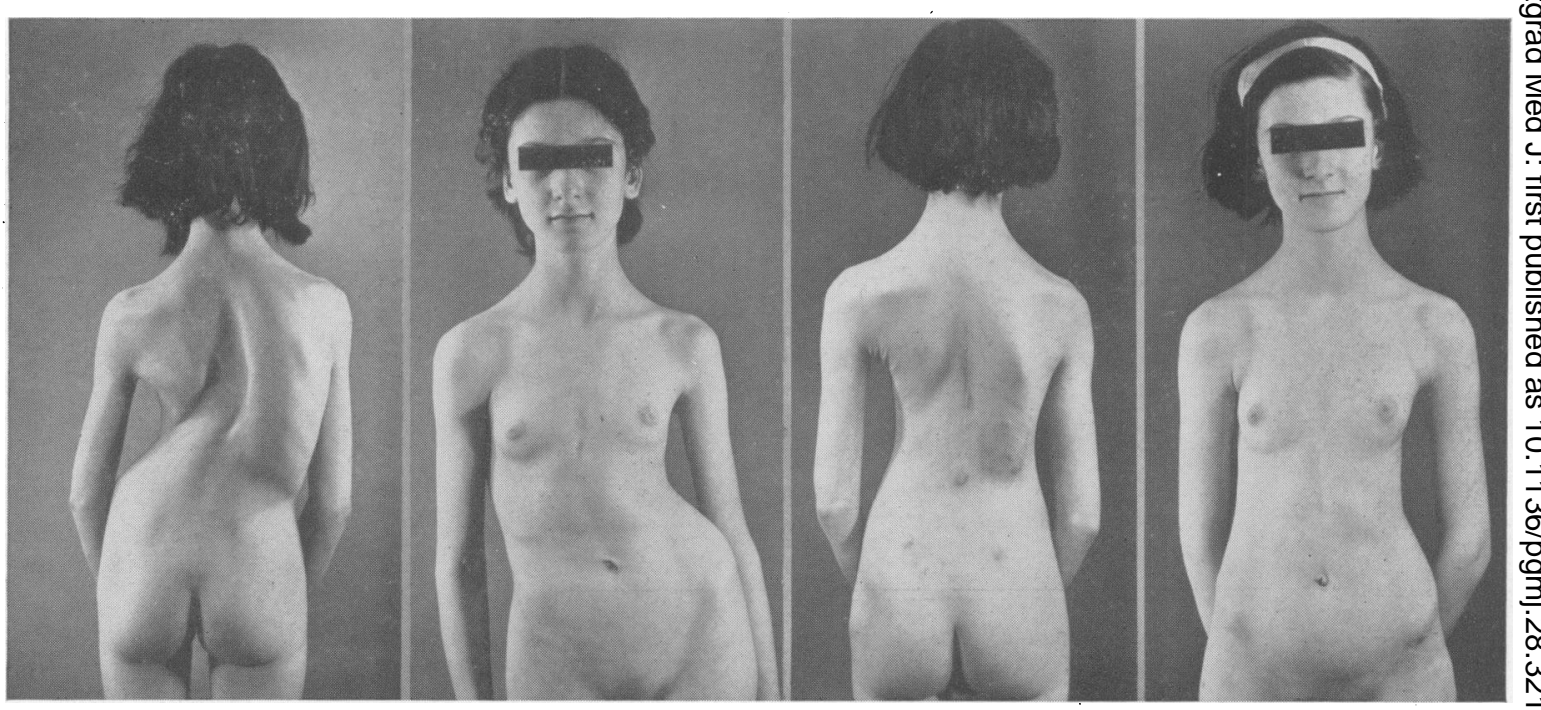

Fig. 12.- A patient with a right idiopathic thoracic scoliosis. The primary curve measured $70^{\circ}$. The final angle after fusion was $45^{\circ}$ (see text).

the fusion is normally the primary curve, though there are a few instances when more than this is necessary. The operation may be done in one, two or even three stages. In the younger child, where periosteal stripping is easy, eight or nine vertebrae may be fused at one stage. The skin incision is straight, as a curved scar accentuates the remaining deformity. Subperiosteal stripping of the spines and laminae and of the articular and transverse processes is performed. The spinous processes are then removed and the laminae and transverse processes are completely decorticated. This gives a deep, wide bed, the floor of which is bleeding cancellous bone. On this base is packed a large mass of chipped bone from the bone bank; a store of refrigerated bone is essential for extensive spinal fusion operations. At the end of the operation silver clips are placed on the spinous process above and below the fusion as radiographic guides.

Blood transfusion is essential. Because a low vital capacity is common in paralytic patients, the prolonged anaesthesia and the post-anaesthetic care may present special problems.

The patients are kept recumbent in the Risser jacket for six months. The spine is supported by a polythene spinal jacket for a further six months, during which time the patient is at home. One year after the operation bending films of the primary curve are taken to exclude pseudarthrosis, and if satisfactory all support is abandoned.

Two cases showing satisfactory results are illustrated in Figs. 12 and 13 . The time-table for correction and fusion of the patient shown in Fig. 12 was as follows:
26.6.50 Admitted Royal National Orthopaedic Hospital, Stanmore. The primary curve measured $70^{\circ}$.

27.6.50 Risser jacket applied.

6.7.50 Hinges and turnbuckle applied.

I 1.7.50 Risser jacket cut transversely.

14.7.50 Correction started.

21.7.50 Small pressure sore; window cut.

4.8.50 Correction from $70^{\circ}$ to $22^{\circ}$.

8.8.50 Plaster filled in.

1 1.8.50 Posterior window cut. Marker film.

15.8.50 One stage fusion T.7 to 12 . Three pints of blood given.

24.8.50 Wound healed, sutures removed.

25.II.50 Appendicectomy.

12.1.5 I Plaster changed. Relapse from $22^{\circ}$ to $34^{\circ}$. 28.2.5 I Cast taken for polythene spinal support.

$20,3,51$ Patient discharged in polythene jacket. Monthly visits to scoliosis clinic.

16.8.5 I Jacket removed. Bending films to right and left showed a variation of only $I^{\circ}$. No evidence of pseudarthrosis.

14.2.52 Final angle is $45^{\circ}$, i.e. correction of $25^{\circ}$ eighteen months after fusion. Iliac apophyses fully developed and further change unlikely.

\section{Complications and Results of Fusion}

Up to date only 50 patients have come to operation out of a total of over 700 under supervision in our clinic. This proportion is low, because so many patients are quite unsuitable for operation at the time of their first visit. The results mentioned here are only part of a preliminary report, because few of the patients operated upon have yet reached maturity.

One death occurred from respiratory failure several hours after operation; the patient was a boy with severe paralytic scoliosis and a low vital capacity. A positive pressure anaesthetic 


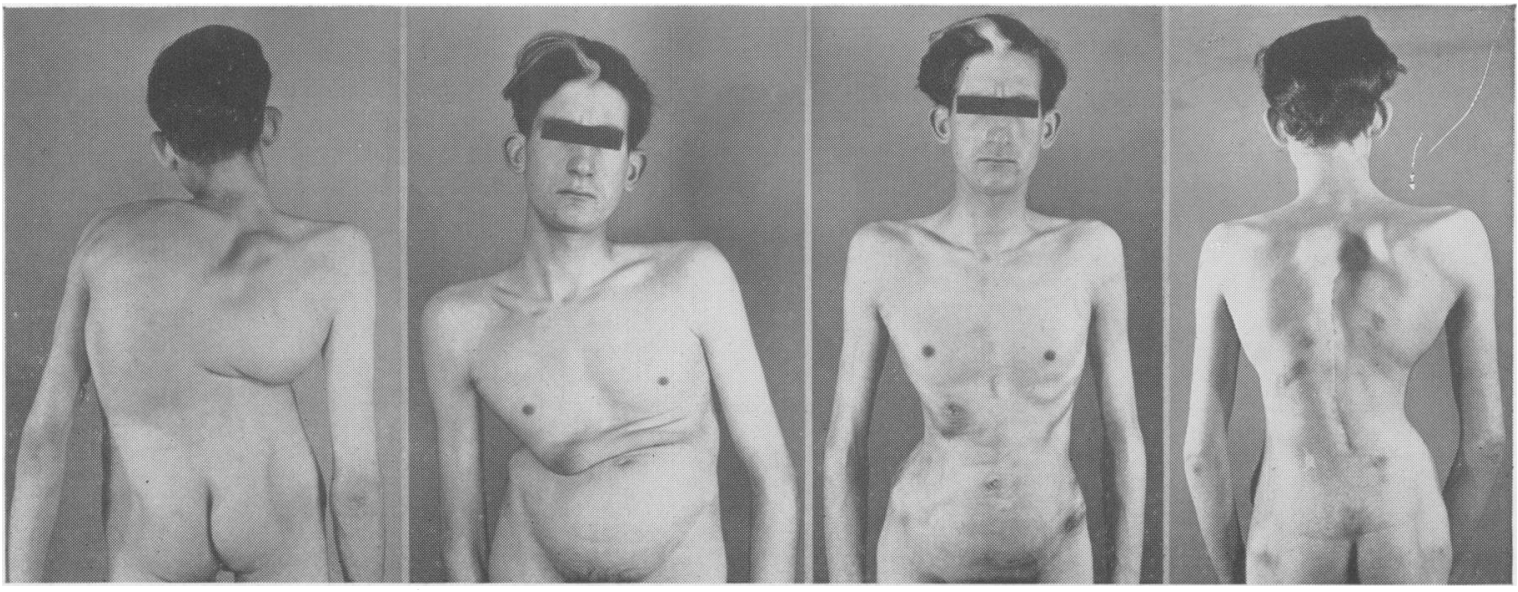

Fig. I3.-An adult patient with a severe paralytic curve secondary to anterior poliomyelitis. The gain in height after correction and fusion was 5 in. Note the altered relation between the right costal margin and the iliac crest. The curve was corrected from $124^{\circ}$ to $78^{\circ}$.

machine is now being used for this type of case. In six cases infection has led to partial loss of the bone graft, but in no case to failure of fusion. The single example of pseudarthrosis visible in the radiographs occurred in a patient with a painful lumbar scoliosis, for which fusion without correction was performed. In four other patients the degree of relapse is such that failure of fusion is suspected, though radiographic evidence of this is lacking.

Correction up to $60^{\circ}$ has been held successfully in several instances. It is true, however, that it is easier to correct such an amount than to hold it, though the relapse is usually only about one quarter to one third of the correction. Apart from the fatality mentioned above, no patient is worse as a result of the operation and no patient has shown an increase of curvature during subsequent growth. This finding is most important, for it is likely that with more accurate prognosis, correction and fusion will in time become more and more preventive of severe deformity, provided of course that the long-term results of fusion remin satisfactory.

\section{Acknowledgment}

Figs. 3, 4, 5 and 8 have been reproduced by kind permission of the Editor of the British Medical Fournal.

\section{BIBLIOGRAPHY}

AMERICAN ORTHOPAEDIC ASSOCIATION RESEARCH COMMITTEE (194I) 'Report on End-Result Study of the Treatment of Idiopathic Scoliosis,' $\mathcal{F}$. Bone $\mathcal{f} t$. Surg., 23, 963 . COBB, J. R. (1948), ' Outline for the Study of Scoliosis,' American Academy of Orthopaedic Surgeons' Instructional Course Lectures, 5, $26 \mathrm{r}$.

FERGUSON, A. B. (1930), 'Study and Treatment of Scoliosis,' Sth. med. F. Bgham., Ala., 23, 116.

JAMES, J. I. P. (195I), ' Two Curve Patterns in Idiopathic Structural Scoliosis,' $\mathcal{~ F . ~ B o n e ~} \mathcal{Y} t$. Surg., 33B, 399.

JAMES, J. I. P. (1951), ' Common Spinal Deformities in Children,' B.M.ं. ., 2, 1270 .

MCELVENNY, R. T. (1941), 'Principles Underlying the Treatment of Scoliosis by Wedging Jacket,' Surg. Gynec. Obstet., 72, 228.

PONSETI, I. V., and FRIEDMAN, B. (1950), 'Prognosis in Idiopathic Scoliosis,' $\mathcal{F}$. Bone Ft. Surg., 32A, $38 \mathrm{r}$.

RISSER, J. C. (1948), 'Important Practical Factors in the Treatment of Scoliosis,' American Academy of Orthopaedic Surgeons' Instructional Course Lectures, $5,248$.

RISSER, J. C., and FERGUSON, A. B. (1936), 'Scoliosis: its Prognosis,' $\mathcal{F}$. Bone 7 t. Surg., 18, 667.

SMITH, A. DE F., BUTTE, F. L., and FERGUSON, A. B. (1938), 'Treatment of Scoliosis by the Wedging Jacket and Spine Fusion.' A Review of 265 Cases, Ibid., 20, 825.

VoM SAAL, F. (1941), 'Management of Scoliosis,' Amer. F. Surg., $52,433$.

VoN LACKUM, W. H. (1948), "The Surgical Treatment of Scoliosis,' American Academy of Orthopaedic Surgeons' Instructional Course Lectures, 5, 236.

\section{RUTHIN CASTLE, NORTH WALES}

A Clinic for the diagnosis and treatment of Internal Diseases (except Mental or Infectious Diseases). The Clinic is provided with a staff of doctors, technicians and nurses.

The surroundings are beautiful. The climate is mild. There is central heating throughout. The annual rainfall is 30.5 inches, that is, less than the average for England.

The Fees are inclusive and vary according to the room occupied.

For particulars apply to THE SECRETARY, Ruthin Castle, North Wales.

Telegrams: Castle, Ruthin.

Telephone: Ruthin 66 